\title{
ESTUDIO DE LA TUTELA COLECTIVA SOBRE DERECHOS SOLIDARIOS
}

\section{ARTÍCULO ORIGINAL}

RIBEIRO, Danilo Miranda ${ }^{1}$

RIBEIRO, Danilo Miranda. Estudio de la tutela colectiva sobre derechos solidarios. Revista Científica Multidisciplinar Núcleo do Conhecimento. Año. 06, Ed. 12, Vol. 02, págs. 152-167. Diciembre 2021. ISSN: 2448-0959, Enlace de acceso: https://www.nucleodoconhecimento.com.br/ley/derechos-solidarios, DOI: 10.32749/nucleodoconhecimento.com.br/ley/derechos-solidarios

\section{RESUMEN}

Este trabajo busca construir un análisis jurídico sobre los elementos del proceso colectivo para la protección de los derechos solidarios en el sistema jurídico brasileño. Debido a que sus disposiciones en las leyes están espaciadas, el estudio de la protección colectiva es el problema de un sistema hermético que es difícil de entender. Con el objetivo de dar paso al estudio del proceso colectivo y descartar este problema, esta investigación tiene como problemática la discusión sobre los principales elementos del proceso colectivo, con el objetivo de demostrar que se trata de un sistema procedimental coherente y armonioso. La metodología utilizada será la revisión bibliográfica. Así, el trabajo presenta como resultado la sistematización de elementos fundamentales para la comprensión de la protección colectiva, al abordar las disposiciones legales y doctrinales sobre la protección de los derechos solidarios, con el fin de abrir el campo para una comprensión de la protección colectiva.

Palabras clave: Proceso Colectivo, Derechos solidarios, Procedimiento Civil, Principios.

\footnotetext{
${ }^{1}$ Magíster en Derechos Humanos y Democracia de la UFPR-Universidad Federal de Paraná. Licenciado en Derecho por la UCSal-Universidad Católica del Salvador. ORCID: https://orcid.org/0000-0001-6503-9625.
}

RC: 103910

Disponible: https://www.nucleodoconhecimento.com.br/ley/derechos-solidarios 


\section{INTRODUCCIÓN}

La protección colectiva es un instrumento procesal esencial para la realización de la justicia social mediante la protección de los bienes jurídicos que no pertenecen sólo a un individuo, sino que conciernen a la colectividad. Resulta que las disposiciones legales sobre el instituto se encuentran en una legislación variada y no prescinden de la comprensión constitucional del asunto, lo que puede ser un obstáculo para la investigación coherente del proceso colectivo. Ante este problema, esta investigación pretende ofrecer una reflexión sobre los elementos esenciales de la protección colectiva de los derechos solidarios, como el concepto de derechos solidarios, la legitimidad y la competencia. Así, el proceso colectivo se presentará de manera coherente y armónica con los principios constitucionales rectores, a fin de eliminar el hermetismo que se puede percibir conociendo la protección colectiva. La metodología utilizada será la revisión bibliográfica de autores que hayan realizado una importante contribución a la comprensión de la protección de los derechos solidarios.

Desde el punto de vista constitucional, el principio del debido proceso legal (art. 5, LIV, CF / 88) debe ser interpretado en su dimensión colectiva, ya que busca reducir las formalidades para lograr un proceso meta individual efectivo y justo. a la jurisdicción. El sistema procesal colectivo atribuye legitimidad a distintas entidades (ope legis), ya que la relevancia de los intereses protegidos evoca la responsabilidad de los distintos actores de asegurar los derechos de una colectividad. En este sentido, el art. 103, del CDC coloca la efectividad erga omnes o ultra partes de las decisiones dictadas en el proceso colectivo (BELLINETTI; SOUZA, 2019).

En cuanto a la clasificación, Zavascki (2005, p. 18) divide los intereses colectivamente en dos categorías. El primero serían intereses individuales homogéneos, que tendrían su carácter colectivo para una cuestión empírica, de contingencia, no necesaria, en la que habría varios sujetos, pero el objeto es divisible. La segunda categoría abarcaría los intereses difusos y colectivos, constituidos por aquellos que son colectivos por su esencia, que se refieren a un 
objeto indivisible y a un número incierto de sujetos. En relación con los intereses solidarios, es posible concebirlos en dos ámbitos separados: derechos individuales homogéneos y derechos colectivos stricto sensu y difusos.

Según Ronaldo Santos (2014), la idea de proteger el derecho colectivo es garantizar la justicia social de los grupos sociales ofendidos o judicializar situaciones que antes no contaban con protección judicial. Para el autor, la acción colectiva fue el antecedente de la Bill of Peace, quese remonta a la historia del siglo XVII:

[...] tratava de uma autorização para o processamento de uma ação individual sob a forma coletiva, concedida nas hipóteses em que o autor requeria que o provimento da demanda englobasse os direitos de todos os indivíduos que estivessem em igual condição de litígio, para que a questão fosse tratada de modo uniforme e com vistas a evitar a multiplicação de processos (SANTOS, 2014, p. 238).

Con un énfasis en la relación de los derechos solidarios como derechos fundamentales, Hachem entiende que los primeros tienen los mismos elementos que estarían relacionados con los derechos fundamentales de las tres generaciones, no solo la tercera, segunda o primera generación:

Por conta da complexidade de sua natureza jurídica e estrutura normativa, todos os direitos fundamentais reúnem concomitantemente a totalidade dos traços que supostamente seriam peculiares a cada uma das gerações: (i) impõem deveres negativos ao Estado; (ii) dirigem ao Poder Público obrigações de fornecer prestações fáticas e normativas; (iii) ostentam a titularidade transindividual alegadamente exclusiva dos "direitos de terceira geração", bem como, simultaneamente, a titularidade individual pretensamente típica dos "direitos de primeira e segunda geração" (HACHEM, 2013, p. 621).

A través de demandas colectivas, se amplifica el acceso a la justicia de una colectividad indeterminada o de un determinado grupo de personas. La socialización y democratización del proceso se señala como beneficios de estas acciones, con la realización de derechos y la alteración de la realidad social; la posibilidad de corrección de un ilícito que no se postularía en una demanda individual ante su pequeño valor particular, pero que colectivamente tiene un gran impacto social; igualdad entre los litigantes; la molecularización de la demanda y la realización del 
principio de seguridad jurídica y confianza legítima frente a decisiones uniformes para casos similares; la reducción de las demandas en el poder judicial, entre muchas otras.

Más específicamente, los intereses individuales homogéneos visten la ropa de los intereses individuales, pero frente a ciertas configuraciones de la sociedad, existe la necesidad de ser protegidos colectivamente. La nomenclatura en sí misma ya traduce parte del concepto de este interés, según la lección de Zavascki:

$\mathrm{Na}$ verdade, a própria denominação interesses individuais homogêneos já exibe dois enunciados: de um lado, trata-se de interesses ontologicamente individuais, não coletivos; de outro lado, que consistem numa multiplicidade de interesses individuais apresentando um determinado grau de uniformidade ou homogeneidade, sua característica mais importante (ZAVASCKI, 2005, p. 18).

Según el artículo 81 de la Ley 8.078/91, los intereses individuales homogéneos se basan en la idea de origen común, en el hecho lesivo que afectó y violó a innumerables personas en su individualidad, sin exigencia de una relación jurídica básica entre los titulares o entre las partes, ni de una unidad de hecho o temporalidad (ZAVASCKI, 2005, p. 21).

Los intereses individuales homogéneos son aquellos que tienen en su origen un hecho nocivo que ha afectado a una colectividad y supera la esfera del interés individual. Por lo tanto, estos derechos tienen un origen común, que surgen en la conducta omisiva omisiva de la parte adversa que afecta a derechos que escapan al mero aspecto individual y crean la pretensión colectiva, llevando a la discusión cuestiones superiores al singular mero lide.

Según la lección de Zavascki (2005, p. 21), son divisibles, apropiadas individualmente, disponibles, transferibles y representadas en los tribunales por el propio titular, episódicamente solidarios, subjetivamente determinadas y directamente reparables. 
Sin embargo, la característica de la individualidad no se aparta del eminente contenido solidario de los derechos individuales homogéneos. Cuando se consideran colectivamente, estos derechos exceden con creces la esfera de interés particular de sus titulares. La característica colectiva no radica en la imposibilidad de ser apropiados individualmente o que sus titulares sean indeterminables, sino en el alcance que revela la violación de la sociedad (ZAVASCKI, 2005, p. 21-22). La lesión a intereses individuales homogéneos viola la paz social y promueve un gran malestar social, lo que genera un efecto sinérgico, ya que ambos se refuerzan.

También sería posible nombrar intereses individuales relevantes o intereses comunes, lo que no se hizo porque la homogeneidad es esencial para la oportunidad de proteger colectivamente la ley (ZAVASCKI, 2005, p. 19).

También en relación con los derechos solidarios, surgen las categorías de derechos difusos y colectivos stricto sensu. En primer lugar, los derechos difusos son aquellos intereses colectivos que son indivisibles, cuyos titulares son indeterminables y no guardan relación jurídica entre ellos (DIDIER JUNIOR; ZANETI JUNIOR, 2014, p. 76-78). El concepto de derechos difusos se encuentra en el análisis del art. 81, I, del Código de Protección al Consumidor, que se conceptualizan como intereses solidarios de carácter indivisible, cuyos titulares son indeterminados y conectados por un hecho común.

Los derechos difusos son aquellos que pertenecen a una colectividad de sujetos que son indeterminados, tienen derechos indivisibles y están vinculados por una situación de hecho (superveniente). Es decir, la relación entre los titulares surgió en un momento posterior al perjuicio, ya que no existía vinculación entre los lesionados en un momento anterior con el hecho que daba causa a la protección colectiva (DIDIER JÚNIOR; ZANETI JÚNIOR, 2014, p. 78).

Luego están los derechos colectivos stricto sensu, que encuentran definición en el art. 81, II de la CDC. Son derechos transindividuales, con la diferencia de que sus titulares son ciertos grupos definidos de personas, es decir, existe una relación jurídica básica entre los titulares, que son indeterminados pero determinables. Es 
decir, la diferencia entre los derechos difusos y los colectivos stricto sensu es que los primeros tienen propiedad indeterminada y los segundos tienen ciertas categorías colectivas como titulares, por lo que se pueden determinar las personas (DIDIER JÚNIOR; ZANETI JÚNIOR, 2014, p. 78-79).

Para los intereses colectivos stricto sensu es esencial identificar qué grupo de individuos fue perjudicado, ya que el derecho es indivisible y los titulares son aquellos que pertenecen a una determinada categoría (DIDIER JÚNIOR; ZANETI JúNIOR, 2014, p. 78).

Es de destacar que la relación jurídica base debe ser anterior a la ocurrencia del hecho lesivo. Es decir, la relación jurídica entre los titulares no proviene del daño, como ocurre en los derechos difusos, sino por la adhesión a la categoría laboral, asociación, cierta sociedad y otras causas que precedieron al perjuicio (DIDIER JÚNIOR; ZANETI JÚNIOR, 2014, p. 78). De esta manera, los titulares del derecho son plenamente determinables y pertenecen a una colectividad que es individualizable.

\section{LEGITIMIDAD}

En situaciones de jurisdicción singular, casi siempre, los temas en cuestión son de interés solo para las partes involucradas en el curso, sin tener ningún peso directo sobre las personas que no están involucradas en el polo pasivo o activo del curso.

Tradicionalmente, la legitimidad activa de la protección del interés individual exige un examen de la propiedad y se remonta al legado del Derecho Romano (DIDIER JÚNIOR; ZANETI JÚNIOR, 2014, p. 79). Por otro lado, el instituto de derecho en nombre de otra persona no es algo mucho más actual, ya que "[...] en la Edad Media, aún no existía el concepto de personalidad jurídica, por lo que ni siquiera era posible considerar que alguien estaba siendo representado por otra persona; la legitimidad ordinaria era la regla absoluta" (ZAVASCKI, 2005, p. 11). 
En el ordenamiento jurídico brasileño, la regla general está prevista en el artículo 18 del Código de Procedimiento Civil, que coloca la titularidad del derecho como requisito fundamental para su discusión, ya que la discusión del derecho que pertenece a la persona de otra persona, con sujeción a los casos expresamente previstos por la ley, permanece.

Sin embargo, además de la legitimidad ordinaria, retratada por el art. 18 del CPC, existe, con carácter excepcional, una legitimidad activa extraordinaria, que se admite en los casos expresamente previstos por la ley, en los que alguien alegará derecho en nombre del titular. En este caso, existe una opción legislativa en los casos en que será posible invocar el derecho de otros ante los tribunales.

Aparte de los supuestos previstos por el cuerpo jurídico, las hipótesis de extraordinaria legitimidad deben considerarse como una consecuencia lógica del ordenamiento jurídico. Neves (2016) es uno de los autores que comentan la posibilidad de una legitimidad extraordinaria cuando no hay permiso legal expreso:

Apesar de o art. 18, caput, do Novo CPC prever que a legitimação depende de autorização expressa da lei, a melhor doutrina entende que, além da previsão legal, também se admite a legitimação extraordinária quando decorrer logicamente do sistema, como acontece com a legitimação recursal do advogado em apelar do capítulo da sentença que versa sobre seus honorários advocatícios (NEVES, 2016, p. 188).

La flexibilidad en relación con la regla del art. 18 del CPC aparece como un mejor molde a la eficacia del instituto, propio de defender los intereses del colectivo. Sin embargo, se advierte: la flexibilización no trae como inevitable seguimiento un procedimiento más simple.

Es notable que se puede garantizar el mismo derecho a personas de diferentes grupos sociales, lo que ocurre con cierta frecuencia, como cuando hay una violación de las garantías sociales. Por lo tanto, existe una dificultad para afirmar que existe una legitimidad para representar a todos los interesados, en vista de la gran diferencia cultural, social o económica que existe entre los titulares de derechos en las demandas colectivas. 
Fue en este contexto (ZAVASCKI, 2005, p. 15) que comenzaron las dificultades para defender los intereses colectivos. Si los individuos son diferentes, ¿cómo se supone que uno de ellos tiene la legitimidad para exigir para todos en defensa de este interés común? ¿Cómo pueden extenderse los efectos de la protección colectiva a quienes no están en los tribunales si no se puede asumir cuál es el interés legítimo de estos otros? ¿Cómo exige que los grandes grupos comparezcan ante los tribunales? ¿Cómo hacer esto si los miembros del grupo ni siquiera están determinados?

Ante la indeterminación de los titulares, una gran diferencia que existe entre ellos y la dificultad práctica de todos para llevar ciertas cuestiones a los tribunales, la legitimación de entidades que tienen como finalidad es la búsqueda y defensa de los intereses de una colectividad.

De esta manera, los colectivos legitimados no son titulares de los derechos que están legitimados para alegar y los titulares de esos derechos tienen la titularidad activa vaciada (NEVES, 2016, p. 189). Por excelencia, en la carta de Zavascki (2005, p. 15), la legitimidad activa en las demandas colectivas podría ser nombrada como la representación de los ausentes.

En la jurisdicción colectiva no existe un vínculo directo entre la titularidad del derecho y la legitimidad, pero sí existe una situación jurídica que aporta la capacidad de ciertas instituciones para defender intereses que no se circunscriben a la idea de individualismo, lo que trae consigo la configuración de una legitimidad extraordinaria. En este sentido:

A legitimação ao processo coletivo é extraordinária: autoriza-se um ente a defender, em juízo, situação jurídica de que é titular um grupo ou uma coletividade. Não há coincidência entre o legitimado e o titular da situação jurídica discutida (DIDIER JUNIOR; ZANETI JUNIOR, 2014, p. 21).

El ordenamiento jurídico brasileño aceptó la opción de asumir la capacidad de legitimación de ciertas instituciones para defender demandas colectivas (ZAVASCKI, 2005, p. 102). Es decir, las legitimadas para efectos de acciones colectivas surgen 
del establecimiento legal, como ocurre en el art. 5 de la Ley de Acción Civil Pública, que enumera al Ministerio Público, a la Defensoría Pública, a la Unión Federal, a los Estados, al Distrito Federal, a los Municipios y demás sujetos, como representantes del colectivo y capaces de interponer una acción civil pública.

En el mismo movimiento de LACP, el artículo 82 del Código de Protección al Consumidor destaca la legitimidad activa del Ministerio Público, la Unión, los Estados y Municipios, los municipios, las empresas públicas, las fundaciones, las sociedades de economía mixta o las asociaciones para defender los derechos homogéneos difusos, colectivos o individuales de los consumidores.

Además, en relación con la legitimidad activa a los efectos de las acciones colectivas, Zavascki (2005, p. 132) plantea un aspecto controvertido que rodea la posibilidad de que el Ministerio Público defiende intereses individuales homogéneos, ya que el CRFB/88 no confiere esta función al parquet.

La lengua vernácula constitucional, en su artículo 129, inciso III, no enumera la defensa de intereses individuales homogéneos como una de las atribuciones del Ministerio Público, que tiene sus actividades vinculadas al texto constitucional. Así, es posible interpretar como una valla jurídica la falta de determinación para defender estos intereses (ZAVASCKI, 2005, p. 132).

Otros abogan por la legitimidad irrestricta del Ministerio Público (ZAVASCKI, 2005, p. 133). Dependiendo del artículo 129, punto IX, del CRFB/88, es función del MP desempeñar funciones compatibles con su finalidad, que incluye la defensa de los intereses sociales e individuales no disponibles, a la luz del art. 127, del CRFB/88. Para esta corriente, los intereses solidarios tienen una presunción de relevancia social, que permite que el parquet actúe (ZAVASCKI, 2005, p. 133).

Finalmente, está la tesis de la legitimidad restringida, que surge como consecuencia de la idea de que el ministerio público tendría legitimidad irrestricta, con la advertencia de que los asuntos de relevancia social sólo podrían ser aquellos que contengan congruencias con las funciones constitucionales del parquet (ZAVASCKI, 
2005, p. 132), esto basado en el art. 127, CRFB/88, sin el esfuerzo de presumir relevancia para poder dar legitimidad al ministerio público.

Sobre la legitimidad del Ministerio Público en la protección de intereses individuales homogéneos, Zavascki concluye que la respuesta a esta pregunta radica a través del análisis de los trajes legales, porque es en la ley donde radica el concepto de interés de relevancia social, lo que asegurará la legitimidad activa del parquet (ZAVASCKI, 2005, p. 132). De esta manera, Zavascki enseña que los intereses de relevancia social están constituidos por: "la imposición de indisponibilidad, la atribución directa de legitimidad al Ministerio Público para su defensa y la caracterización expresa de relevancia social" (ZAVASCKI, 2005, p. 136).

La división es indiscutible. A falta del aspecto de relevancia social que retrata Zavascki, no es necesario hablar de la legitimidad del parquet para actuar en defensa de intereses individuales homogéneos.

Finalmente, cabe destacar las características que Didier revela en la legitimación colectiva activa, como regulación por el artículo 5 de la Ley Federal № 7.347/85 y el art. 82 del CDC, siendo porteado por entidades que no actúan en nombre propio, ya que los titulares no tienen capacidad postulatoria:

Enfim, o certo é que a legitimação coletiva possui as seguintes características: a) está regulada, inicialmente, por lei (art. 5ำ da Lei Federal no 7.347/85; art. 82 do CDC etc.); b) é conferida a entes públicos, privados e despersonalizados, e, até, ao cidadão, na ação popular; c) o legitimado coletivo atua em nome próprio na defesa de direitos que pertencem a um agrupamento humano (pessoas indeterminadas, comunidade, coletividade ou g rupo de pessoas, titulares de direitos individuais abstratamente considerados, na forma do a1i. 81 do CDC e seus incisos); d) esse agrupamento humano não tem personalidade judiciária, portanto não pode atuar em juízo para proteger os seus direitos, 26 cuja defesa cabe aos legitimados coletivos, que possuem legitimação autônoma, exclusiva e concorrente e simples (DIDIER JUNIOR; ZANETI JUNIOR, 2014, p. 212).

RC: 103910

Disponible: https://www.nucleodoconhecimento.com.br/ley/derechos-solidarios 


\section{COMPETENCIA}

La competencia es un elemento fundamental para la satisfacción del debido proceso. La defensa de los derechos solidarios se produce en favor de una colectividad, que puede separarse y sin ningún vínculo que la una, lo que llama la atención sobre el momento de fijación de competencia.

Para la fijación de la competencia territorial en las demandas colectivas, Neves enumera siete pasos necesarios para la divulgación de la sentencia competente. Se destaca que la fijación de la jurisdicción territorial es consecuencia del análisis de la legislación procesal, del mismo modo que se hace en los casos de protección singular, ya que se trata de una materia vinculada a la ley tratada de forma isonómica a la protección individual (NEVES, 2016, p. 168).

Sin embargo, es necesario destacar que la legislación que regula la protección de los derechos colectivos puede establecer la jurisdicción originada en ciertos tribunales u órganos atípicos, en la forma de lo que pueda ocurrir en el proceso colectivo especial, la acción popular, la acción civil pública, el requerimiento colectivo, la orden colectiva y la acción de falta administrativa.

Debido a la relevancia de las medidas, vale la pena señalar que la competencia en el ámbito colectivo está regulada por las artes. 2, la Ley de Acción Civil Pública, y 93, del Código de Protección al Consumidor, que establecen como criterio de competencia territorial el lugar del daño.

Además, dependiendo de la legislación del LACP, la competencia sería territorial y funcional, lo que plantea el debate de si la competencia se debería a los foros, a la cuestión territorial, o a la mayor eficacia y facilidad, en virtud del criterio funcional. Sin embargo, es cierto que la jurisdicción territorial es absoluta, lo que lleva a su respeto obligatorio.

Tras determinar el foro competente, se inicia la búsqueda de la sentencia competente, que se hará desde las leyes de organización del poder judicial y del 
CPC. Esto se debe a que la jurisdicción de la sentencia se ve afectada por la jurisdicción territorial, nunca al revés (NEVES, 2016, p. 186).

\section{EJECUCIÓN DE LA SENTENCIA DICTADA EN ACCIONES COLECTIVAS}

La base para la ejecución de una sentencia individual está desgastada por el cumplimiento de una sentencia colectiva, que debe tener lugar como continuación del proceso de conocimiento, después del momento en que el deudor no ha cumplido con la orden de la sentencia. La fase de ejecución se cumplirá mediante el cumplimiento de una sentencia, según las circunstancias previstas en el art. 515 del CPC.

En relación con los derechos difusos y colectivos stricto sensu la protección del derecho material se realizará a través de la ejecución colectiva, que se desarrollará en beneficio de la comunidad, lo que se hará a través de los aspectos utilizados en la ejecución individual, sin ninguna especialidad procesal por el carácter colectivo de interés (NEVES, 2016, p. 392-393).

De la ejecución puede surgir un amparo (prohibición de ilegalidad) o reparatorio in natura, como reversión de daños y perjuicios, o en efectivo, que, en este caso, debe ser revertido a la persona jurídica de derecho público que enfrentó el perjuicio o al Fondo de Derechos Difusos (art. 13 de la Ley Federal № 7.347/1985) (DIDIER JÚNIOR; ZANETI JÚNIOR, 2014, p. 412).

En cuanto a la legitimidad, cualquier parte integrante del polo activo podrá promover la ejecución, aunque no haya participado en la fase de cognición, según la interpretación del artículo 15 de la Ley 7.347, que enumera como requisito el lapso de tiempo de 60 días del tránsito final para que nuevos sujetos participen en el proceso a efectos de iniciar la etapa de implementación (DIDIER JÚNIOR; ZANETI JÚNIOR, 2014, p. 413). Es decir, la ejecución colectiva de derechos difusos o colectivos stricto sensu puede hacerse por cualquier extraordinaria legitimación, 
respetando el curso temporal del tránsito final. Esto también está previsto en el art. 15 de la CBPC-IBDP y en el art. 26 de la CBPC-UERJ/UNESA.

Se enfatiza la obligación de proponer el cumplimiento de la sentencia como atribución esencial al parquet, en el cuidado de los intereses colectivos (NEVES, 2016, p. 391).

Sin embargo, vale la pena distinguir que la obligación del Ministerio Público de ejecutar una sentencia en acción civil pública surge después de 60 días de la sentencia definitiva de la sentencia, según el art. 15, de la Ley 7.347/85.

En relación con la acción popular, queda pendiente el deber del Ministerio Público de ejecutar la sentencia cuando el ciudadano se encuentre inerte por más de 60 días, contados a partir de la publicación de la sentencia de segundo grado, según el art. 16, de la Ley 4.717/65. Es decir, existiría el deber funcional del parquet móvil de cumplir con una sentencia provisional, ante la posibilidad de modificación del comando de sentencia.

Por lo tanto, también existe la posibilidad de aplicación individual en la ejecución de los derechos difusos y colectivos stricto sensu, según la lección de Didier:

É também possível que a sentença proferida em um processo em
que se discutem direitos difusos ou coletivos seja utilizada por um
indivíduo corno título de uma execução individual, tendo em vista a
extensão in utilibus da coisa julgada coletiva ao plano individual, já
examinada. Obviamente, antes de executar a decisão, o indivíduo
deverá proceder à liquidação do seu crédito, em que deverá
demonstrar, inclusive, que é titular de um crédito (liquidação com
thema decidendum mais amplo do que a liquidação individual
prevista no CPC, restrita à decisão sobre o valor ou sobre a coisa a
ser executada) (DIDIER JUNIOR; ZANETI JUNIOR, 2014, p. 415).

En cuanto a los derechos individuales homogéneos, que son derechos individuales agregados por su origen común, la ejecución se llevará a cabo individualmente por los titulares. Es decir, el fuerte componente de colectividad que existía en la fase de conocimiento se ralentizó en la fase de ejecución, ya que el interés individual será tratado como colectivo en la fase de ejecución (NEVES, 2016, p. 394). En cuanto a 
los foros, el artículo 516 del CPC impone una regla estricta, un criterio de competencia absoluta, que llevaría al cumplimiento de una sentencia para la sentencia que resolvió el caso en primer grado de jurisdicción.

La interpretación de esa disposición legal incluye la ejecución individual de una sentencia colectiva, sin embargo, por criterios de practicidad Neves (2016, p. 376) defiende como competente para la resolución individual de la sentencia colectiva el foro del domicilio del síndico, de modo que no exista una gran concentración de procedimientos en el distrito que resolvió el caso en primer grado de jurisdicción y menos carga para el exequente.

Sin embargo, cabe señalar que existió un veto presidencial en relación con el párrafo único del art. 97 del CDC, lo que excluye la hipótesis de que el foro de la ejecución de una sentencia colectiva se elija como el del domicilio del exequente.

Según Zavascki (2005, p. 164), no hay forma de considerar inútil el veto, ya que abre el camino a la interpretación y revela razones que deben ser consideradas, ya que son consideradas por los legisladores.

En vista de la determinación expresa de la competencia para la ejecución de la sentencia, es la de la sentencia que juzgó el caso en primer grado de jurisdicción, según el art. 516, II del CPC, no existen dudas sobre la competencia.

Finalmente, hay un punto polémico en el art. 98 del CDC, en relación a la posibilidad del Ministerio Público y los organismos públicos (legitimados del art. 82 del CDC) de promover la ejecución de intereses individuales homogéneos, ya que la esfera colectiva se vacía al momento de la ejecución de intereses individuales. Destacando la lección de Zavascki:

Quanto ao Ministério Público e órgãos públicos, não se vislumbra circunstância na qual tais entes teriam algum interesse em promover liquidação e execução específicas em favor de particulares, ainda mais mediante representação. Em consequência, é certo não possuir - Ministério Público legitimidade para promover o tratamento individualizado da sentença genérica, mas não porque ele não tem poderes para agir em nome próprio em favor das vítimas e 
sucessores e sim porque, nessa hipótese, não se trata de defesa de interesses sociais ou individuais indisponíveis (ZAVASCKI, 2005, p. 163).

Finalmente, es interesante observar que incluso quienes no forman parte del proceso pueden quejarse de lo propuesto en una sentencia colectiva, ya que su propósito es dar concreción a los derechos de la comunidad:

\begin{abstract}
Diante disso, os instrumentos processuais utilizados para tutela dos interesses transindividuais devem ser adaptados à natureza do interesse substancial em questão, de modo a lhe proporcionar uma maior efetividade. Para tanto, algumas alterações legislativas foram feitas com a finalidade de atender esse escopo. Dentre elas menciona-se a tratativa dada à sentença coletiva, à coisa julgada e à execução de sentença coletiva. A sentença coletiva, dada sua natureza, possui um alcance diferenciado no que se refere aos indivíduos por ela albergados, podendo atingir, inclusive, aquele que não foi parte no processo, dada a extensão secundum eventum litis da coisa julgada coletiva ao plano individual (ALFREDO, BALEOTI, 2012, p. 435).
\end{abstract}

\title{
5. GUARDIANES DE LA URGENCIA EN LAS ACCIONES COLECTIVAS
}

En general, la protección de la urgencia en las acciones colectivas no presenta divergencia de razonamiento en relación con las acciones individuales (DIDIER JÚNIOR; ZANETI JÚNIOR, 2014, p. 342), que trae incidencia de CPC cuando existen los requisitos establecidos en su art. 300.

Sin embargo, existen ciertos procedimientos especiales que prevén esta técnica procesal, como el art. 12 del LACP, que permite otorgar protección temprana en la defensa de ciertos intereses colectivos (DIDIER JÚNIOR; ZANETI JÚNIOR, 2014, p. 342).

A modo de ejemplo, también, el art. 4 del LACP prevé la posibilidad de medidas cautelares con el alcance de prevenir daños a los bienes jurídicos descritos en el mismo. Acertadamente, Didier conduce a la corrección de lo dispuesto en dicho artículo, ya que la medida sería satisfactoria, ya que otorgaría el derecho buscado mediante la provocación de la potestad judicial, no una medida cautelar: 
Embora mencione expressamente a tutela cautelar, a redação do dispositivo não dá margem a dúvida: não se trata de tutela cautelar, mas, sim, tutela inibitória, que é satisfativa e visa exatamente obter providência judicial que impeça a prática de ato ilícito e, por consequência, a ocorrência de um dano (DIDIER JÚNIOR; ZANETI JÚNIOR, 2014, p. 343).

Es importante destacar ciertos impedimentos para el otorgamiento de protección provisional en las acciones colectivas, como las disposiciones del artículo 2 de la Ley Federal № 8.437/1992 y el $\S 2$ del art. 22 de la Ley $N^{\circ} 12.016 / 2009$, que limitan el otorgamiento de un requerimiento judicial en una orden de seguridad colectiva 0 acción civil pública a la audiencia del representante judicial de la persona jurídica de derecho público, deberán realizarse en un plazo máximo de 72 horas.

Asimismo, es similar a lo que ocurre para el otorgamiento de un amparo en el conflicto colectivo por la posesión de bienes, el cual está condicionado al cumplimiento de los requisitos establecidos en el art. 565 del Código procesal civil.

\section{CORREGIBILIDAD EN ACCIONES COLECTIVAS}

No existe un medio recursal específico para su uso en acciones colectivas. Lo que hay menciones excepcionales de la repetibilidad en la aplicación en demandas colectivas, lo que trae aplicación general del sistema recursal del Código de Procedimiento Civil (NEVES, 2016, p. 324)

Con respecto a la impugnación de una decisión de interlocución, el artículo 1.015 del CPC enumera la lista de hipótesis que abren alcance al daño del instrumento. Dependiendo de la autorización expresa de este artículo, en su párrafo XIII, otros casos previstos por la ley permiten la gestión del daño del instrumento, en cuyo artículo 19, §1, de la Ley 4.717/65, con la disposición de que es apropiado agravar el instrumento debido a una decisión de interlocución en el contexto de la acción popular.

Según Neves (NEVES, 2016, p. 325), debido al microsistema colectivo, la norma antes mencionada debería ser aplicable en todas las demás acciones colectivas, lo 
que permitiría la interposición de un instrumento de daño en cualquier decisión interlocutoria que surgiera en un proceso colectivo.

Con respecto a la legitimidad recursiva, es interesante analizar que existen excepciones a la regla del 996 del CPC, que establece a la parte perdedora, a los terceros interesados y a los fiscales como activos legítimos de los recursos.

Cabe destacar que el artículo 996 del CPC trata del interés recursivo y no de la legitimidad recursal, ya que, al tratarse de una parte perdedora, se realiza un análisis de sucumbir, lo que lleva a un análisis concreto de la decisión que cuestiona el interés recursal (NEVES, 2016, p. 330). La legitimidad no tiene nada que ver con el interés, porque es una parte legítima recurrir al que gana o pierde, el criterio de establecer la legitimidad es totalmente independiente del contenido de la decisión.

Vale la pena destacar un tema importante: de acuerdo con la regla del artículo $19, \S$ 2 , de la Ley 4.717/65, cualquier ciudadano puede apelar las sentencias y decisiones dictadas a raíz de la acción popular. Resulta que, en función de la norma establecida en el artículo 996 del CPC, el ciudadano que no compone el polo activo del curso no encaja como tercero o tercero sin éxito, ya que el derecho de comentario en la acción popular es difuso, lo que elimina el concepto de tercero interesado en el párrafo único del art. 996, CPC. Así, el ciudadano no compone la lista de sujetos que tienen legitimidad recursiva, sino que encuentra permiso en el LAP para apelar.

En cuanto a los efectos recursivos (abstinentes, traslativos, expansivos, sustitutivos, regresivos y diferidos) no hay particularidad cuando se generan en el proceso colectivo. Sólo en cuanto al efecto suspensivo, es necesario examinar la legislación relativa a cada acción, con el fin de verificar la ausencia o presencia de cada efecto recursivo mencionado (NEVES, 2016, p. 332).

Cabe destacar la legislación reguladora de la Acción Civil Pública, que otorga al juez un efecto suspensivo en los recursos con el alcance de perjudicar irreparablemente a las partes, en función del art. 14 de la Ley 7.347/1985, que conformó el entendimiento de que los recursos interpuestos en el ámbito de la Acción Civil 
Pública no tienen efecto suspensivo únicamente por su mera interposición (NEVES, 2016, p. 332). Así, el recurso, que, por regla general, tiene efectos suspensivos, en virtud del artículo 1.012 de la Ley de Enjuiciamiento Civil, debe cumplir con los requisitos enumerados en el art. 14 del LACP (evitar daños irreparables aparte) para evitar que la sentencia dictada en Acción Civil Pública genere efectos inmediatos.

\section{CONSIDERACIONES FINALES}

En vista de la discusión llevada a cabo por este trabajo, es posible observar que el texto del CRFB/88 gana fuerza y eficacia con la protección de los derechos colectivos, a la manera de una búsqueda de la materialización de las garantías sociales que están previstas en la lengua vernácula constitucional y pueden ser reclamadas judicialmente.

Tal instrumento es extremadamente interesante para que la justicia social se efectúe en una sociedad de masas, donde la violación de un derecho puede ir más allá de la esfera individual y llegar a una colectividad de personas. Con esta razón en mente, para responder al problema presentado inicialmente, se destacó el sesgo constitucional de garantizar los derechos solidarios al conferir singularidad a las disposiciones legales, que buscan asegurar los derechos colectivos en confluencia con el logro del principio de justicia social y debido proceso.

Al explorar los elementos esenciales de la protección colectiva, se presentó un sistema armónico que encuentra coherencia para garantizar la protección del bien jurídico de toda la colectividad. Así, se superó el problema del hermetismo de este sistema procesal, ya que se abordó de tal manera que se discutiera su matriz constitucional y sus fundamentos jurídicos. Por lo tanto, lo que podría parecerse a un sistema desconectado, porque tiene estandarización en diversas legislaciones, se interpreta como un instrumento integrado que se mueve para garantizar derechos que pertenecen no solo a una persona, sino a la sociedad. 


\section{REFERENCIAS}

ALFREDO, Luciana Romaneli Rodrigues e BALEOTTI, Francisco Emílio. A sentença coletiva e a efetividade dos direitos transindividuais. Revista Jurídica Cesumar, Vol. 12, No 2, 2012.

BELLINETTI, Luiz Fernando; SOUZA, Raíssa Fabris. Obstáculos para a efetividade da tutela de direitos transindividuais por entidades sindicais em prejuízo ao efetivo acesso à justiça. Revista Cidadania e Acesso à Justiça, Florianópolis, SC, v. 5, p. 1-17, 2019.

DIDIER JUNIOR, Fredie; ZANETI JUNIOR, Hermes. Curso de Direito Processual Civil: processo coletivo. 9. ed. Salvador: JusPodivm, 2014.

HACHEM, Daniel Wunder. A dupla titularidade (individual e transindividual) dos direitos fundamentais e econômicos, sociais, culturais e ambientais. Revista de Direitos Fundamentais e Democracia, v. 14, n. 14, Curitiba, p. 618-688, jul./dez. 2013.

NEVES, Daniel Amorim Assumpção. Manual de processo coletivo. São Paulo: Método, 2016.

SANTOS, Enoque Ribeiro dos. Processo coletivo do trabalho. 2. ed., rev., atual. e ampl. Rio de Janeiro: Forense, 2018.

ZAVASCKI, Teori Albino. Processo coletivo: tutela de direitos coletivos e tutela coletiva de direitos. Tese (Doutorado Programa de Pós-graduação em Direito) Faculdade de Direito da Universidade Federal do Rio Grande do Sul, Porto Alegre, 2005.

Enviado: Diciembre de 2021.

Aprobado: Diciembre de 2021. 